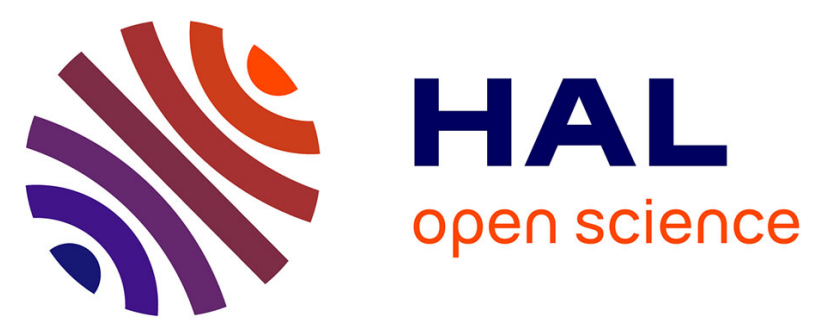

\title{
Interleukin-10 overexpression does not synergize with the neuroprotective action of RGD-containing vectors after postnatal brain excitotoxicity but modulates the main inflammatory cell responses.
}

Pau Gonzalez, Hugo Peluffo, Laia Acarin, Antonio Villaverde, Berta Gonzalez, Bernardo Castellano

\section{To cite this version:}

Pau Gonzalez, Hugo Peluffo, Laia Acarin, Antonio Villaverde, Berta Gonzalez, et al.. Interleukin-10 overexpression does not synergize with the neuroprotective action of RGD-containing vectors after postnatal brain excitotoxicity but modulates the main inflammatory cell responses.. Journal of Neuroscience Research, 2012, 90 (1), pp.143-59. 10.1002/jnr.22741 • pasteur-00686279

\section{HAL Id: pasteur-00686279}

https://hal-riip.archives-ouvertes.fr/pasteur-00686279

Submitted on 9 Apr 2012

HAL is a multi-disciplinary open access archive for the deposit and dissemination of scientific research documents, whether they are published or not. The documents may come from teaching and research institutions in France or abroad, or from public or private research centers.
L'archive ouverte pluridisciplinaire HAL, est destinée au dépôt et à la diffusion de documents scientifiques de niveau recherche, publiés ou non, émanant des établissements d'enseignement et de recherche français ou étrangers, des laboratoires publics ou privés. 


\section{IL-10 OVEREXPRESSION DOES NOT SYNERGIZE WITH THE NEUROPROTECTIVE ACTION OF RGD- CONTAINING VECTORS AFTER POSTNATAL BRAIN EXCITOTOXICITY, BUT MODULATES THE MAIN INFLAMMATORY CELL RESPONSES}

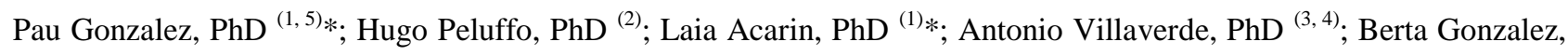
$\mathrm{PhD}^{(1)}$ and Bernardo Castellano, $\mathrm{PhD}^{(1)}$.

${ }^{1}$ Unit of Medical Histology, Department of Cell Biology, Physiology and Immunology and Neuroscience Institute, Autonomous University of Barcelona, Spain.

${ }^{2}$ Neurodegeneration Laboratory, Pasteur Institute of Montevideo and Department of Histology and Embryology, Faculty of Medicine, UDELAR, Uruguay.

${ }^{3}$ Institute of Biology and Biomedicine and Department of Genetics and Microbiology from the Autonomous University of Barcelona, Spain.

${ }^{4}$ Networking Biomedical Research Center in Bioengineering, Biomaterials and Nanomedicine (CIBER-BBN), Spain

${ }^{5}$ Molecular Neurology Laboratory, National Paraplegic Hospital of Toledo, Spain

\section{RUNNING TITLE: EFFECTS OF IL-10 OVEREXPRESSION IN IMMATURE BRAIN}

*Corresponding authors.

Medical Histology, Torre M5, Department of Cell Biology, Physiology and Immunology, Autonomous University of Barcelona, 08193 Bellaterra (Barcelona), Spain. Tel: 34935811826, Fax: 34935812392, e-mail: laia.acarin@uab.cat, paug@ sescam.jccm.es.

Supported by: BFU2005-02783 from the Ministry of Science and Innovation, Government of Spain and 061710 from the Marato TV3 Foundation to BG; BFU2008-04407/BFI from the Ministry of Science and Innovation, Government of Spain to BC and ACI2009-0910 from the Ministry of Science and Innovation, Government of Spain to AV. 


\begin{abstract}
Anti-inflammatory cytokines such as IL-10 have been used to modulate and terminate inflammation and provide neuroprotection. Recently, we reported that the modular recombinant transfection vector NLSCt is an efficient tool for transgene overexpression in vivo, which induces neuroprotection due to its RGD-mediated integrin interacting capacity. We here aimed to evaluate the putative synergic neuroprotective action exerted by IL-10 overexpression using NLSCt as transfection vector after an excitotoxic injury to the postnatal rat brain. For this purpose, lesion volume, neurodegeneration, astroglial and microglial responses, neutrophil infiltration and pro-inflammatory cytokine production were analyzed at several survival times after intracortical NMDA injection in postnatal day 9 rats followed by injection of NLSCt combined with the IL-10 gene, a control transgene or saline vehicle solution. Our results show no combined neuroprotective effect between RGD-interacting vectors and IL-10 gene therapy, instead IL-10 overexpression using NLSCt as transfection vector increased lesion volume and neuronal degeneration at 12 hours and 3 days post-lesion. In parallel, NLSCt/IL-10 treated animals displayed increased density of neutrophils and microglia/macrophages, and a reduced astroglial content of GFAP and vimentin. Moreover, NLSCt/IL-10 treated animals did not show any variation in interleukin-1 $\beta$ and tumour necrosis factor $\alpha$ expression but a slight increase in interleukin-6 content at 7 days pot-lesion. In conclusion, overexpression of IL-10 by using NLSCt transfection vector did not synergistically neuroprotect the excitotoxically damaged postnatal rat brain, but induced changes in the glial and inflammatory cell response.
\end{abstract}

Key words: IL-10, gene therapy, brain, postnatal, excitotoxicity 


\section{BACKGROUND}

CNS inflammation is characterized by cellular responses including astroglial and microglial reactivity, leukocyte infiltration, and increased production of different inflammatory-related molecules such as pro-inflammatory cytokines, chemokines and free radical producing enzymes. Overall, these responses lead to an exacerbation in damage severity. To modulate and terminate the progression of inflammation, the expression of anti-inflammatory endogenous molecules is upregulated after CNS damage. Accordingly, interleukin-10 (IL-10), a cytokine with a strong antiinflammatory nature (Moore et al. 2001; Strle et al. 2001), is upregulated in CNS pathologies such as multiple sclerosis (Hulshof et al. 2002) and in different experimental models of CNS injury including experimental autoimmune encephalomyelitis (EAE) (Jander et al. 1998; Ledeboer et al. 2003), middle cerebral artery occlusion (MCAO) (Zhai et al. 1997), traumatic brain injury (TBI) (Kamm et al. 2006) and a genetic model of Alzheimer disease (Apelt and Schliebs 2001). Noteworthy, as shown after MCAO, expression of pro-inflammatory molecules decreases when IL-10 is upregulated (Zhai et al. 1997). In agreement, the lack of IL-10 in knockout mice results in an elevated production of pro-inflammatory cytokines after endotoxic shock (Agnello et al. 2000), and several in vivo and in vitro experimental studies have reported the inhibitory role exerted by IL-10 administration on inflammatory processes such as astroglial and microglial reactivity (Balasingam et al. 1994; Balasingam and Yong 1996; Jackson et al. 2005; Mesples et al. 2003; Ooboshi et al. 2005; Pang et al. 2005), leukocyte infiltration (Knoblach and Faden 1998) and production of chemokines (Guo et al. 1998), pro-inflammatory cytokines (Benveniste et al. 1995; Knoblach and Faden 1998; Ledeboer et al. 2000; Molina-Holgado et al. 2001b; Ooboshi et al. 2005; Pousset et al. 2001; Sawada et al. 1999) and inflammatory-related enzymes (Molina-Holgado et al. 2002a; Molina-Holgado et al. 2002b; Molina-Holgado et al. 2001a). As a consequence, numerous experimental studies have focused on the neuroprotective potential of IL-10 administration during the course of different CNS pathologies achieving, in many cases, promising results (Brewer et al. 1999; Dietrich et al. 1999; Jackson et al. 2005; Knoblach and Faden 1998; Ooboshi et al. 2005; Spera et al. 1998).

However, only a few of the aforementioned studies have approached the actions exerted by IL-10 in the injured immature CNS. This lack of information becomes especially important when considering that the modulation of the inflammatory response associated to developmental brain damage constitutes a promising therapeutical approach (Acarin et al. 2001; Arvin et al. 2002; Cai et al. 2004; Chew et al. 2006; Cornette 2004; Dammann and Leviton 1997; Fan et al. 2005; Ferriero et al. 1996; Froen et al. 2002; Galasso et al. 2000; Gonzalez and Ferriero 2008; Hamada et al. 1994; Kadhim et al. 2001; Kremlev et al. 2007; Leviton and Dammann 2004; Lin et al. 2006; Mesples et al. 2003; Silverstein et al. 1997; Tahraoui et al. 2001; Tsuji et al. 2000), and that the immature brain displays distinct physiological and morphological features, as a consequence of its ongoing postnatal development, that determine its particular response to brain damage (Ferriero 2004; Vannucci and Hagberg 2004), including a higher susceptibility to inflammation-mediated damage and an exacerbation of the brain's inflammatory response associated to injury 
(Dammann and Leviton 1997; Lawson and Perry 1995; Rezaie and Dean 2002). In this regard, we have recently shown that IL-10 and its receptor are upregulated in glial cells in the excitotoxically injured postnatal brain (Gonzalez et al. 2009), and that their increase temporally correlates with a decrease in the expression of pro-inflammatory molecules such as interleukin-1 beta (IL-1 $\beta)$, tumor necrosis factor alpha (TNF $\alpha)$, cyclooxygenase-2 (COX-2) and inducible nitric oxide synthase (iNOS) (Acarin et al. 2000; Acarin et al. 2002), strongly supporting that IL-10 overexpression, alone or in combination with other therapies, may constitute an anti-inflammatory and putatively protective therapy for immature brain injury.

In order to modulate post-injury processes, we have demonstrated that the modular protein-based transfection vector NLSCt is an efficient tool for transgene overexpression after developmental excitotoxic damage (Peluffo et al. 2006; Peluffo et al. 2003). Each $\beta$-galactosidase subunit of the tetrameric NLSCt accommodates a polylysine tail with DNA condensing/attaching properties, a nuclear localization motif, and an integrin interacting domain obtained from the GH loop region of the foot-and-mouth disease virus, displaying a prototypical three-amino acid Arg-Gly-Asp (RGD) sequence, showing cell attachment and internalization properties (Aris and Villaverde 2003). As we previously shown, intracerebral injection of NLSCt combined with a transgene is able to induce the expression of the carried transgene after excitotoxic injury in neurons, microglia and astrocytes throughout the damaged tissue for at least 3 days post injection (Peluffo et al. 2003). Interestingly, NLSCt is not only effective as a transfection vector for in vivo gene delivery, but it also induces neuroprotection mediated by its RGD integrin interacting capacity (Peluffo et al. 2006; Peluffo et al. 2007). Taking advantage of this special feature of NLSCt, we have reported that the combined use of NLSCt together with the overexpression of transgenic $\mathrm{Cu} / \mathrm{Zn}$ superoxide dismutase produced an additive response, increasing neuroprotection to excitotoxicity (Peluffo et al. 2005). As the neuroprotective effect of RGD-interaction was dependent on glial cells in vitro, and induced an early increase in microglial reactivity (Peluffo et al. 2006; Peluffo et al. 2007), we hypothesized that the introduction of a glial modulatory gene such as IL-10, would provide a more effective neuroprotective tool. Therefore, the aim of the present study was to evaluate the putative synergy between the NLSCt mediated neuroprotection and the potential anti-inflammatory and neuroprotective effects of IL-10 overexpression in the excitotoxically damaged postnatal rat brain. 


\section{MATERIALS AND METHODS}

\section{Animals and excitotoxic lesions.}

All experimental animal work was conducted according to Spanish regulations following European Union directives. The ethical commission of the Autonomous University of Barcelona approved all experimental procedures. All efforts were made to minimize animal suffering in every step.

For this study, we used a total of 115 nine-day old (P9) Black Hooded Long Evans postnatal rats (15-20 gr) (Janvier, France) of both sexes (litter size was cut down to 10 on P0 and, in all cases, litters were composed by a similar number of males and females). Excitotoxic lesions were performed as previously described (Acarin et al. 1996; Acarin et al. 1997). Briefly, animals were placed in a stereotaxic frame adapted for newborns (Kopf) under isoflurane anesthesia (Veterinaria Esteve, Barcelona, Spain), the skull was opened using a drill and $0.15 \mu \mathrm{L}$ of saline solution $(0.9 \% \mathrm{NaCl}, \mathrm{pH} 7.4)$ containing 18.5 nmols of N-methyl-D-aspartate (NMDA) (Sigma, Steinheim, Germany) were injected into the right sensorimotor cortex at the level of the coronal suture, at $2 \mathrm{~mm}$ lateral to bregma and $0.5 \mathrm{~mm}$ of depth. After suture, pups were placed on a thermal pad and maintained at normothermia during 1 hour before being returned to the dam.

\section{Treatment paradigm and experimental groups.}

NLSCt was produced and purified from Escherichia coli as previously detailed (Aris and Villaverde 2003). Plasmid DNA containing the IL-10 gene (pORF5/mIL10) (Invitrogen, Paisley, UK) was purified from DH5 $\alpha$. coli bacterial strain using "Wizard Plus Minipreps: DNA purification system” (Promega, Madison, WI) following supplier protocol. Complex formation between transfection vector and plasmidic DNA was induced by co-incubation of NLSCt $(0.8 \mu \mathrm{g} / \mu \mathrm{L})$ with the IL-10 plasmid $(0.024 \mu \mathrm{g} / \mu \mathrm{L})$ in saline solution at room temperature (RT) for one hour before injection.

Two hours after NMDA injection, pups were anaesthetized again and injected with $1 \mu \mathrm{L}$ of NLSCt transfection vector (Peluffo et al. 2006) complexed to the plasmid containing the IL-10 gene, at the same stereotaxic coordinates used for NMDA administration. After suture, pups were placed again in a thermal pad and maintained at normothermia for 1 hour before being returned to the dam. Along this study, these animals will be referred as NMDA/NLSCt/IL-10. In addition, a set of NMDA lesioned animals were injected with either $1 \mu \mathrm{L}$ of NLSCt complexed with a plasmid containing the GFP control gene (pEGFP-C1) (Clontech, Mountain View, CA) or $1 \mu \mathrm{L}$ of vehicle saline solution. These animals were used as control groups and will be referred as NMDA/NLSCt/GFP and NMDA/SAL, respectively.

\section{Experimental groups and sample processing.}

According to the treatment received and the survival time, animals were distributed in different groups and subgroups as indicated in Table I. For histological and immunohistochemical analysis, rats were intracardially perfused with $4 \%$ paraformaldehyde (Sigma, Steinheim, Germany) in $0.1 \mathrm{M}$ phosphate buffer. Brains were removed, postfixed 
for 4 hours in the same fixative, cryoprotected in $30 \%$ sucrose, frozen with $\mathrm{CO}_{2}$ and stored at $-80{ }^{\circ} \mathrm{C}$. Frozen brains were cut with the aid of a cryostat (Leica microsystems, Nussloch, Germany) and parallel $30 \mu \mathrm{m}$ thick sections were obtained and stored either mounted on gelatin-coated slides or as free floating sections in Olmos antifreeze buffer at -20 ${ }^{\circ} \mathrm{C}$. For protein quantification by ELISA assays, pups were sacrificed by decapitation. Ipsilateral brain hemispheres were immediately removed, frozen with liquid nitrogen and stored at $-80{ }^{\circ} \mathrm{C}$ until homogenization in a potter homogenizer in ice-cold Tris-50mM/EDTA-1mM buffer containing a cocktail of protease (Roche, Manheim, Germany) and phosphatase inhibitors (Sigma, Steinheim, Germany). Finally, for IL-10 expression analysis by quantitative Real Time PCR, pups were killed by decapitation and ipsilateral brain hemispheres were immediately removed, frozen in liquid nitrogen and stored at $-80^{\circ} \mathrm{C}$ until RNA isolation.

\section{Histology and immunohistochemistry.}

Nissl staining. For lesion volume quantification, a set of parallel sections mounted on gelatin-coated slides from each animal was stained following the Nissl method. Briefly, sections were incubated with $0.1 \%$ toluidine blue (Panreac, Barcelona, Spain) in Wallpole buffer at room temperature for 5 minutes and washed with distilled water. Sections were dehydrated, cleared in xylene (Panreac, Barcelona, Spain) and coverslipped with DPX (Panreac, Barcelona, Spain).

Fluoro-Jade $B(F J B)$ staining. In order to visualize degenerating neurons, sections were processed for FJB labeling. Briefly, after hydration in decreasing graded ethanol, sections were rinsed in water and oxidized with $0.06 \%$ potassium permanganate $\left(\mathrm{MnO}_{4} \mathrm{~K}\right)$ (Panreac, Barcelona, Spain) for 15 minutes. Then, sections were rinsed in distilled water and immersed for 20 minutes in $0.0004 \%$ FJB (Histo-Chem Inc, Jefferson, AK) plus $1 \%$ glacial acetic acid (Panreac, Barcelona, Spain) solution. Finally, sections were air dried, dehydrated and coverslipped with DPX (Panreac, Barcelona, Spain).

Tomato Lectin Histochemistry. To visualize microglia/macrophages, sections were processed for tomato lectin histochemistry (Acarin et al. 1994). Briefly, endogenous peroxidase was blocked by incubating slides for 10 minutes in $2 \%$ hydrogen peroxide $\left(\mathrm{H}_{2} \mathrm{O}_{2}\right)$ (Sigma, Steinheim, Germany) diluted in $70 \%$ methanol (VWR Prolabo, Briare, France) solution. Sections were then rinsed and incubated for $2 \mathrm{~h}$ at $37^{\circ} \mathrm{C}$ in the biotinylated tomato lectin (TL) (Lycopersicon esculentum) (Sigma, Steinheim, Germany) in a 1:150 dilution. After washes, sections were incubated for 1 hour at RT with HRP-linked avidin (Dako, Glostrup, Denmark) diluted to 1:200. The peroxidase reaction product was visualized by incubating sections in $100 \mathrm{~mL}$ of Tris buffer containing $50 \mathrm{mg}$ of 3-diaminobenzidine (DAB) (Sigma, Steinheim, Germany) and $33 \mu \mathrm{L} \mathrm{H}_{2} \mathrm{O}_{2}$ (Sigma, Steinheim, Germany). Finally, sections were dehydrated, cleared in xylene (Panreac, Barcelona, Spain) and coverslipped with DPX (Panreac, Barcelona, Spain).

Glial Fibrillary Acidic Protein (GFAP), vimentin and myeloperoxidase (MPO) immunohistochemistry. In order to visualize astrocytes, GFAP and vimentin immunohistochemistry were performed; whereas, MPO 
immunohistochemistry was carried out for neutrophil demonstration. Briefly, three sets of parallel sections mounted on gelatin-coated slides from each animal were processed for endogenous peroxidase blockade as detailed in the previous paragraph. Subsequently, sections were treated with buffer blocking (BB) containing 10\% fetal calf serum (FCS) (Invitrogen, Paisley, UK) in Tris-buffered saline (pH 7.4) with 1\% Triton X-100 (Sigma, Steinheim, Germany) for 1 h. Then, sections were incubated overnight at $4{ }^{\circ} \mathrm{C}$ plus $1 \mathrm{~h}$ at RT in one of the following primary antibodies: rabbit antiGFAP (Dako, Glostrup, Denmark), mouse anti-vimentin (Dako, Glostrup, Denmark) or rabbit anti-MPO (Dako, Glostrup, Denmark) diluted to 1:500, 1:1000 and 1:400 in BB, respectively. After washes, sections were incubated for 1h in either HRP-linked anti-rabbit (GE Healthcare, Buckinghamshire, UK) or HRP-linked anti-mouse (GE Healthcare, Buckinghamshire, UK) secondary antibodies diluted to 1:200. The peroxidase reaction product was visualized using the DAB method as detailed before for TL histochemistry. Finally, sections were dehydrated, cleared in xylene (Panreac, Barcelona, Spain) and coverslipped with DPX (Panreac, Barcelona, Spain).

CD11b/phospho-histone H3 double immunohistochemistry. To evaluate microglia/macrophage proliferation, double immunofluorescence was carried out, in a set of free floating sections from a representative animal per experimental group and survival time, by combining CD11b (microglia/macrophage marker) and phospho-histone H3 (p-H3) (cell division marker). To accomplish that, sections were first incubated with mouse anti-CD11b primary antibody (Serotec, Oxford, UK) (1:1000) and subsequently with Cy2-conjugated anti-mouse secondary antibody (GE Healthcare, Buckinghamshire, UK) (1:1000). Samples were further processed by using rabbit anti-p-H3 primary antibody (Upstate, Temecula, CA) (1:3000) followed by incubation with Cy3-conjugated anti-rabbit (GE Healthcare, Buckinghamshire, UK) (1:1000). Finally, sections were dehydrated, cleared in xylene (Panreac, Barcelona, Spain) and coverslipped with DPX (Panreac, Barcelona, Spain).

\section{Cytokine ELISA assays.}

IL-1 $\beta$, TNF $\alpha$ and IL-6 protein concentrations were evaluated in ipsilateral damaged hemispheres by using the following commercially available kits: Interleukin-1 $\beta[(\mathrm{r}) \mathrm{IL}-1 \beta]$ rat ELISA system, Tumour Necrosis Factor Alpha [(r)TNF $\alpha$ ] Rat Biotrak ELISA system and Interleukin-6 [(r)IL-6] rat ELISA system, according to the manufacturer's instructions (GE Healthcare, Buckinghamshire, UK). Total protein concentration of each sample was obtained by using the bicincholinic acid method (Smith et al. 1985).

\section{RNA isolation, cDNA synthesis and Real Time PCR.}

RNA was isolated from frozen ipsilateral damaged brain hemispheres by using the Total RNA isolation system Kit (Promega, Madison, WI). Concentration and purity of mRNA were determined by measuring absorbance at 260 and $280 \mathrm{~nm}$ and by electrophoresis. Moreover, in order to eliminate putative genomic DNA traces, further digestion with turbo-DNase (Applied Biosystems, Foster city, CA) was performed following the supplier protocol. DNA digestion was carried out at $37^{\circ} \mathrm{C}$ for $45 \mathrm{~min}$ followed by enzyme deactivation at $70^{\circ} \mathrm{C}$ for $15 \mathrm{~min}$. 
Two micrograms of mRNA were retrotranscribed, as described by the provider, by using the High Capacity cDNA Reverse Transcription kit (Applied Biosystems, Foster city, CA) with random hexamers (Applied Biosystems, Foster city, CA) for transgenic IL-10 and with specific oligonucleotide primers for glyceraldehyde-3-phosphatedehydrogenase (GAPDH) (Table II). Retrotranscription was performed at $42{ }^{\circ} \mathrm{C}$ for 45 min, followed by deactivation of the enzyme at $95^{\circ} \mathrm{C}$ for $5 \mathrm{~min}$ and $4{ }^{\circ} \mathrm{C}$ for $5 \mathrm{~min}$.

To perform GAPDH quantitative Real Time PCR, the SYBR Green PCR Core reagents kit was used (Applied Biosystems, Foster city, CA). Amplification of cDNA was carried out in MicroAmp Optical 384-wells Reaction Plates (Applied Biosystems, Foster city, CA) on an ABI PRISM 7900HT Sequence Detection System (Applied Biosystems, Foster city, CA). The reaction mixture $(10 \mu \mathrm{L})$ was composed of SYBR Green buffer, $3 \mathrm{mM}$ of $\mathrm{MgCl}_{2}, 875 \mu \mathrm{M}$ of dNTPs with dUTP, 0.3 U of AmpliTaq Gold, 0.12 U of Amperase UNG, 7.5 pmol of each primer (Table II), 12.5 ng of cDNA and nuclease-free $\mathrm{H}_{2} \mathrm{O}$. The reaction conditions for cDNA amplification were 2 min at $50{ }^{\circ} \mathrm{C}$ and 10 min at $95^{\circ} \mathrm{C}$ followed by 40 cycles of 15 seconds at $95^{\circ} \mathrm{C}$ and $1 \mathrm{~min}$ at $59^{\circ} \mathrm{C}$. In order to carry out IL-10 Real Time PCR, we used the specific TaqMan Gene Expression Assay (Applied Biosystems, Foster city, CA) for murine IL-10, following supplier instructions. Amplification of cDNA was performed in MicroAmp Optical 96-wells Reaction Plates (Applied Biosystems, Foster city, CA) on an ABI PRISM 7900HT Sequence Detection System (Applied Biosystems, Foster city, CA). In this case, cDNA was preamplified by initial $10 \mathrm{~min}$ at $95^{\circ} \mathrm{C}$ followed by 15 cycles of 15 seconds at $95^{\circ} \mathrm{C}$ and 1 min at $60{ }^{\circ} \mathrm{C}$. Finally, the amplification reaction was performed by initial $10 \mathrm{~min}$ at $95^{\circ} \mathrm{C}$ followed by 40 cycles of 15 seconds at $95{ }^{\circ} \mathrm{C}$ and $1 \mathrm{~min}$ at $60^{\circ} \mathrm{C}$. IL-10 mRNA expression was quantified with reference to the housekeeping gene

GAPDH as: $2^{\text {(Threshold cycle of target mRNA-Threshold cycle of GAPDH mRNA) }}$

\section{Lesion volume, densitometrical analysis and cell counting.}

For lesion volume quantification, twenty two parallel Nissl stained sections (from bregma -1.8 mm to bregma $+4.5 \mathrm{~mm}$ ), separated by $300 \mu \mathrm{m}$ and containing the whole lesion volume, were analyzed for each animal as previously described (Peluffo et al. 2006). In each section, lesioned area and total ipsilateral hemispheric area were measured by using analySIS 3.2 software (Soft Imaging System, Münster, Germany).

As NLSCt-induced transgene overexpression after postnatal excitotoxic injury is restricted to the lesioned nervous parenchyma (Peluffo et al. 2003), all densitometrical and cell count analysis were specifically performed in the lesioned areas. For densitometrical analysis, three sections from each animal corresponding to $-0.3,0$ and $+0.3 \mathrm{~mm}$ from bregma were selected and 20x images were taken at the center of the lesioned ipsilateral hemisphere as well as the corresponding contralateral non-lesioned area (Fig. 1). Images were analyzed by using analySIS 3.2 software (Soft Imaging System, Münster, Germany). The "immunoreactivity grade" was calculated as previously described (Acarin et al. 1997; Acarin et al. 1999b) for GFAP, vimentin and TL. In all cases, for each animal, values were obtained as the mean of three sections. 
Quantification of microglia/macrophages and neutrophil density was performed in the same 20x micrographs $\left(\right.$ area $\left.=0.18 \mathrm{~mm}^{2}\right)$ used for densitometrical analysis (Fig. 1). To estimate the density of degenerating neurons, a set of two images/section $\left(40 \mathrm{x}\right.$, area $\left.=0.04 \mathrm{~mm}^{2}\right)$ from the damaged tissue surrounding the central lesion core and from the same sections detailed above were used (Fig. 1). Images used for cell density evaluation were processed by using the tools for cell counting in AnalySIS 3.2 software (Soft Imaging System, Münster, Germany). Briefly, threshold was set qualitatively according to the immunohistochemical signal in contralateral control hemisphere, and it was maintained unchanged for all images. Subsequently, images were binarized and processed by the application of a particle separation filter. Finally, number of particles were measured in each image by using the analyze particles tool.

\section{Statistical analysis.}

In all cases, statistical analysis was performed using Statview 5.0.1 software and differences among groups were evaluated by means of one-way ANOVA followed by Fisher post-hoc test. All parameters were presented as mean values \pm SEM and $\mathrm{p}<0.05$ was considered statistically significant. 


\section{RESULTS}

NMDA injection into the sensorimotor cortex of the P9 immature rat brain, as observed in the NMDA/SAL control group, induced tissue damage, neuronal degeneration and glial response affecting the ipsilateral sensorimotor cortex, corpus callosum, striatum and hippocampus, as we have previously described in detail (Acarin et al. 1999a; Acarin et al. 1999b). However, differences in lesion volume, neuronal death, astroglial and microglial reactivity, neutrophil infiltration and pro-inflammatory cytokine expression were observed in NMDA/NLSCt/IL-10 and NMDA/NLSCt/GFP animals when compared with the NMDA/SAL control group.

As we have shown in a recent publication (CITA), where a detailed characterization of IL-10 mRNA and protein expression pattern in the normal and excitotoxically injured postnatal rat brain is given, intracortical NMDA injection led to an increase in IL-10 gene expression at $72 \mathrm{hpl}$ when compared to non lesioned control animals, while no differences were observed at any other evaluated survival time (10, 24 and $48 \mathrm{hpl}$, and $7 \mathrm{dpl})$. Therefore, we here aimed to evaluate if NLSCt carrying the IL-10 containing plasmid was able to increase IL-10 expression. Quantification of IL10 expression by Real Time PCR showed that, at 24 hpl, NMDA/NLSCt/IL-10 animals displayed a significant increase in IL-10 mRNA content as compared to both NMDA/NLSCt/GFP and NMDA/SAL groups (Fig. 2).

\section{Lesion volume and neuronal cell death.}

Evaluation of injured tissue volume on Nissl stained sections (Fig. 3A) showed that, at 12 hpl (Fig.3B), NMDA/NLSCt/IL-10 animals displayed a significantly higher lesion volume than control NMDA/SAL and NMDA/NLSCt/GFP groups, while no differences were observed between NMDA/SAL and NMDA/NLSCt/GFP groups. By 72 hpl (Fig. 3B), NMDA/NLSCt/GFP animals exhibited a significant reduction in lesion size when compared to the NMDA/SAL control group. Noteworthy, at this time point, NMDA/NLSCt/IL-10 animals did not show significant differences when compared to NMDA/SAL control animals, but displayed a significant higher lesion volume than the NMDA/NLSCt/GFP group. At 7 dpl (Fig. 3B), no differences in lesion size were detected between the three experimental groups, although a trend towards higher lesion volume can be found in animals that overexpressed IL-10 (NMDA/NLSCt/IL-10 vs NMDA/SAL, $\mathrm{p}=0.09 ;$ NMDA/NLSCt/IL-10 vs NMDA/NLSCt/GFP, $\mathrm{p}=0.06$ ).

Microscopic analysis of FJB staining revealed that neuronal degeneration was more pronounced in NMDA/NLSCt/IL-10 animals than in both the NMDA/NLSCt/GFP and the NMDA/SAL control groups (Fig. 4). These qualitative observations were confirmed by cell counting, which demonstrated that the density of FJB+ degenerating neurons at $12 \mathrm{hpl}$ was significantly higher in the NMDA/NLSCt/IL-10 group than in the other two groups (Fig. 4A, BD). By 72 hpl, NMDA/NLSCt/GFP treated animals displayed an evident reduction in the density of FJB+ degenerating neurons in comparison with the NMDA/SAL control group, while NMDA/NLSCt/IL-10 animals did not differ from the NMDA/SAL controls, showing a significantly higher density of FJB+ degenerating neurons than NMDA/NLSCt/GFP animals (Fig. 4A, E-G). Finally, at 7 dpl, no differences between the three groups were observed (Fig. 4A, H-J). 


\section{Microglial response.}

Analysis of sections processed for tomato lectin (TL) histochemistry to evaluate the microglia/macrophage response (Fig. 5) showed that, at $12 \mathrm{hpl}$, TL+ microglia/macrophages already exhibited a round amoeboid profile in all three experimental groups. In NMDA/NLSCt/GFP and NMDA/NLSCt/IL-10 groups, an increase in the density of TL+ microglia/macrophages was seen in the lesioned cortex in comparison with NMDA/SAL control animals (Fig. 5C-E). In agreement, densitometrical analysis and cell counts showed that, at this time point, NMDA/NLSCt/GFP and NMDA/NLSCt/IL-10 animals exhibited a significantly higher immunoreactivity grade (Fig. 5A) and increased density of TL+ microglia/macrophages (Fig. 5B). By $72 \mathrm{hpl}$, both round and poorly ramified microglial cells were observed in all groups, but NMDA/NLSCt/IL-10 animals showed a robust increase in the immunoreactivity grade and in the density of TL+ microglia/macrophages as compared to both control groups, which were not different from each other (Fig. 5A, B, F-H). At 7 dpl, all groups displayed TL+ cells mostly poorly ramified (Fig. 5I-K), and no significant differences between groups were detected by densitometrical or cell count analysis. Finally, in all groups, and as shown by double immunofluorescence, we found that only a low percentage of CD11b+ microglia/macrophages were labeled with the proliferation marker $\mathrm{pH} 3$ at the different survival times (Fig. 6).

\section{Astroglial response.}

Analysis of the astroglial response by GFAP and vimentin immunohistochemistry (Fig 7 and 8 ) showed no signs of astroglial reactivity in lesioned areas by $12 \mathrm{hpl}$ in any of the three experimental groups. However, by $72 \mathrm{hpl}$, all groups exhibited a significant increase in the immunoreactivity grade when compared to the previous survival time (Fig. 7A, B). As previously reported (Acarin et al. 1999b), astroglial reactivity was restricted to the areas of tissue damage and characterized by an increase in the thickness and length of astroglial processes. Notably, at 72 hpl, NMDA/NLSCt/GFP treatment did not induce variations in astroglial reactivity in comparison with NMDA/SAL control group, while in NMDA/NLSCt/IL-10 animals, GFAP and vimentin immunoreactivity was significantly lower than that observed in control groups (Fig. 7A, B) (Fig. 8A-C and G-I). By 7 dpl, all groups displayed, throughout the whole lesioned parenchyma, maximum astroglial reactivity characterized by an evident increase in the presence of reactive astrocytes, which showed thicker and longer processes and an increase in GFAP and vimentin immunoreactivity grades (Fig. 8D-F and J-L, respectively). No differences between groups were found (Fig. 7A, B).

\section{Neutrophil recruitment.}

In all three groups, analysis of MPO immunohistochemistry for the visualization of neutrophils was carried out at $12 \mathrm{hpl}$, when maximum neutrophil infiltration occurs in this experimental paradigm (L. Acarin, unpublished observations).. Our results demonstrated the presence of small round shaped MPO+ cells with highly stained intracellular granules distributed throughout the whole lesioned parenchyma, but showing higher concentration in the lesion center. Noticeably, MPO+ cell density estimation showed that, when compared to the NMDA/NLSCt/GFP and 
the NMDA/SAL groups, NMDA/NLSCt/IL-10 animals exhibited a significant increase in the density of MPO+ cells (Fig. 9).

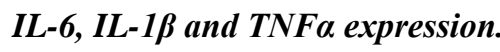

As we previously demonstrated (CITA), when compared to saline injected control animals, NMDA injection in the postnatal rat brain increased IL-1 $\beta, \mathrm{TNF} \alpha$ and IL-6 protein expression between 10 and $24 \mathrm{hpl}$, and at $7 \mathrm{dpl}$ respectively. No temporal differences were detected in these parameters in saline injected control animals at all analyzed survival times. We therefore sought to determine the existence of potential differences in the expression of pro-inflammatory cytokines between the three experimental groups used to perform the present study. Quantification of cytokine content by ELISA assays in ipsilateral damaged hemispheres showed that all cytokines analyzed were present in all three groups and survival times (Fig.10). IL-1 $\beta$ concentration was maximal at $12 \mathrm{hpl}$ in all groups, when NMDA/NLSCt/GFP and NMDA/NLSCt/IL-10 groups exhibited a significant increase in IL-1 $\beta$ content in comparison with the NMDA/SAL control group. At $72 \mathrm{hpl}$ and $7 \mathrm{dpl}$, all experimental groups displayed significantly decreased IL$1 \beta$ content, and no differences between groups were detected. TNF $\alpha$ expression also reached maximum levels at $12 \mathrm{hpl}$ in all experimental groups, but no significant differences between treatments were found at any of the evaluated time points. Finally, although no differences between groups were detected in IL-6 concentration at 12 and $72 \mathrm{hpl}$, at $7 \mathrm{dpl}$, when maximum IL-6 content is observed in all groups, NMDA/NLSCt/IL-10 animals displayed a slight increase in IL-6 when compared to either the NMDA/NLSCt/GFP or the NMDA/SAL group. 


\section{DISCUSSION}

Our results show the neuroprotective effect of the NLSCt transfection vector, conjugated with the GFP control transgene, after postnatal excitotoxic injury, as previously demonstrated (Peluffo et al. 2006; Peluffo et al. 2007). This neuroprotective effect is attributed to the RGD integrin interacting domain of NLSCt (Peluffo et al. 2007), and is accompanied by an early increase in the microglia/macrophage response. In fact, RGD-mediated neuroprotection is mediated by a glial dependent mechanism, and it is reliant on the presence of glial cells in cell cultures (Peluffo et al. 2007).

In addition, and diverging from our initial hypothesis based on studies demonstrating IL-10 neuroprotection in different models of acute CNS injury (Brewer et al. 1999; Dietrich et al. 1999; Froen et al. 2002; Jackson et al. 2005; Knoblach and Faden 1998; Mesples et al. 2003; Ooboshi et al. 2005; Pang et al. 2005; Rodts-Palenik et al. 2004; Spera et al. 1998), treatment with NLSCt combined with IL-10 overexpression did not induce further neuroprotection than that observed by the administration of NLSCt carrying the control transgene. On the contrary, at $12 \mathrm{hpl}$ and $3 \mathrm{dpl}$, a significant increase in lesion volume and neurodegeneration was observed in lesioned animals treated with IL-10.

\section{Dual effect of IL-10 overexpression on lesion progression in the immature versus the adult brain.}

Although it has been widely reported that IL-10 may induce neuroprotection in different models of acute CNS injuries (Brewer et al. 1999; Dietrich et al. 1999; Froen et al. 2002; Jackson et al. 2005; Knoblach and Faden 1998; Mesples et al. 2003; Ooboshi et al. 2005; Pang et al. 2005; Rodts-Palenik et al. 2004; Spera et al. 1998), conflicting results, based on different experimental approaches, have been also reported (Brewer et al. 1999; Knoblach and Faden 1998; Mesples et al. 2003). In this sense, systemic administration of IL-10 is neuroprotective in different injury paradigms (Brewer et al. 1999; Froen et al. 2002; Knoblach and Faden 1998; Mesples et al. 2003; Pang et al. 2005; Rodts-Palenik et al. 2004), while intraparenchymatic administration can either be neuroprotective (Dietrich et al. 1999; Jackson et al. 2005; Ooboshi et al. 2005; Spera et al. 1998), provide no effect in lesion outcome (Knoblach and Faden 1998; Mesples et al. 2003), or even be deleterious for lesion progression (Brewer et al. 1999). Notably, age is a crucial factor determining IL-10-mediated effects after acute CNS injuries as, in contrast to the adult, the postnatal brain exhibits a deficient response to this anti-inflammatory cytokine. In agreement, neither IL-10 gene deletion in IL-10 knockout mouse, or intracerebral IL-10 administration showed no effect in lesion development after neonatal mouse brain excitotoxicity (Mesples et al. 2003). Importantly, Mesples and colleagues show that intracerebral IL-10 administration at the time of excitotoxic lesion induction, showed a non-significant dose-dependent tendency to increase lesion size, which was counteracted by anti-IL-10 antibody administration (Mesples et al. 2003). This attenuated effect could be explained by the fact that IL-10R expression is very low in the excitotoxically lesioned neonatal rat brain until $48 \mathrm{hpl}$ (Gonzalez et al. 2009). Accordingly, in the present study, IL-10 overexpression follows the temporal pattern of IL-10R expression in this postnatal excitotoxic lesion model (Gonzalez et al. 2009), demonstrating that IL-10 
overexpression significantly increased lesion volume and neurodegeneration, even in the context of a neuroprotective transfection vector, further demonstrating the differential response of the immature brain to this anti-inflammatory cytokine. This deleterious effect of anti-inflammatory cytokines during immaturity has also been reported after TGF- $\beta$ administration, which increases damage severity in a model of postnatal ibotenate-mediated excitotoxic brain injury (Mesples et al. 2005), whereas it is neuroprotective after adult brain damage (Buisson et al. 2003). Altogether, these observations support the hypothesis that the injured immature brain responds particularly to anti-inflammatory based approaches (Northington 2006), highlighting the need to generate specific neuroprotective strategies for the neonatal brain.

Although we do not have a conclusive explanation for the detrimental effect of IL-10 overexpression in our experimental paradigm, our results show that IL-10 overexpression, by means of the neuroprotective transfection vector NLSCt, also modulates inflammatory processes, increasing leukocyte infiltration and unbalancing the astroglial and microglial responses after postnatal excitotoxicity, as will be discussed below.

\section{IL-10 overexpression increased neutrophil infiltration.}

Correlating with the IL-10-mediated increase in lesion volume and neurodegeneration we observed, at $12 \mathrm{hpl}$, an increase in MPO+ cells in the lesioned tissue with the morphological characteristics of neutrophils, the main cell type expressing the enzyme MPO at this early post-lesion survival time after stroke or excitotoxicity (Breckwoldt et al. 2008). Previous reports have described neutrophil accumulation after acute damage to the postnatal CNS (Hudome et al. 1997; Lawson and Perry 1995; Palmer et al. 2004), which are thought to induce injury exacerbation by reducing the microvascular flow and releasing cytotoxic molecules (Hartl et al. 1996; Kochanek and Hallenbeck 1992). In agreement, experimental interventions aiming to reduce polymorphonuclear cell infiltration induced neuroprotection after acute immature brain injury (Hudome et al. 1997; Palmer et al. 2004). Noticeably, NLSCt treatment itself did not change post-lesion neutrophils recruitment (Peluffo et al. 2007), suggesting a role of IL-10. However, while the increase in neutrophil density seems to correlate with IL-10-induced exacerbation of tissue damage reported here, we do not have any explanation to clarify the IL-10-mediated effect on neutrophil infiltration, as it is established that IL-10 inhibits the production of several CXC chemokines in immune cells (Moore et al. 2001), and specifically, IL-8 production in microglial cultures (Ehrlich et al. 1998). Moreover, previous reports in the damaged adult brain have demonstrated that IL-10 inhibits neutrophil infiltration (Abraham et al. 2004), further supporting the idea that age-dependent differences may account for this unexpected effect of IL-10.

\section{IL-10 overexpression reduced astroglial reactivity.}

As previously reported in other injury paradigms, another effect of IL-10 overexpression is the astroglial reduction in GFAP and vimentin immunoreactivity (Balasingam and Yong 1996; Pang et al. 2005; Woiciechowsky et al. 2004). In contrast, NLSCt administration itself did not modulate astroglial reactivity, suggesting that the reduction in 
astroglial GFAP and vimentin depends on overexpressed IL-10, probably mediated by a direct mechanism, as we have recently shown that reactive astrocytes are the main cell type expressing the IL-10 receptor (IL-10R) in this experimental lesion model (Gonzalez et al. 2009). Noteworthy, correlation of reduced astrogliosis and damage exacerbation has been previously reported by using genetic ablation of astroglial intermediate filaments, which leads to increased acute brain damage severity (Nawashiro et al. 2000; Nawashiro et al. 1998), and has been related to reduced astroglial capacity to respond to hypo-osmotic stress, regenerate the compromised blood brain barrier or undergo glutamate uptake (Anderova et al. 2001; Hughes et al. 2004; Pekny et al. 1998). In the same way, potentially neuroprotective functions of astroglial response include neuronal trophic and metabolic support, oxygen free radicals detoxification by producing antioxidant enzymes, or secretion of critical factors involved in proper angiogenesis and revascularization (Liberto et al. 2004), further supporting the putative correlation between reduced astroglial reactivity and increased lesion volume and neurodegeneration.

\section{NLSCt induced early activated microglia, but IL-10 overexpression elicited a sustained microglial activation.}

It is interesting to note that while the neuroprotective effect of NLSCt treatment is accompanied by an early increase in the microglia/macrophage response, the neurotoxicity observed after IL-10 overexpression correlates with a sustained exacerbation of the microglial response until $3 \mathrm{dpl}$, suggesting that early microglial activation, but not sustained, can be related to neuroprotection. Both an increase in endogenous microglial proliferation or increased recruitment of peripheral monocytes could be the cause of the IL-10-mediated increase in microglia/macrophages at 3 dpl. In this regard, our colocalization studies between the proliferation marker $\mathrm{pH} 3$ and microglial cells showed no apparent differences in $\mathrm{pH} 3$ immunostaining between the groups at $12 \mathrm{hpl}$ and 3 dpl, implying that microglia/macrophage increase could be related to monocyte infiltration. However, although this hypothesis may well correlate with the IL-10-induced rise in neutrophil recruitment reported here, IL-10 is known to inhibit leukocyte infiltration to the damaged adult brain (Abraham et al. 2004; Kline et al. 2002), and reduce the adhesion capacity of monocytes and lymphocytes to endothelial cells in vitro (Krakauer 1995; Mostafa Mtairag et al. 2001), probably by inhibiting expression of adhesion molecules and chemokines (Buchwald et al. 1999; Chen and Manning 1996; Guo et al. 1998; Krakauer 1995). In this regard, although IL-10 effects on the microglia/macrophage response may constitute a direct effect of IL-10, as this cell population expresses IL-10R (Gonzalez et al. 2009); an indirect effect mediated by the reduction in the astroglial response may also have a prominent role. It should be noted that the ablation of proliferating reactive astrocytes in different models of acute injury induces an evident increase in the number of infiltrated leukocytes and phagocytic cells (Bush et al. 1999; Faulkner et al. 2004; Myer et al. 2006).

It should also be mentioned that, in contrast to our findings, it is established that IL-10 inhibits microglial activation, both in vitro (O'Keefe et al. 1999; Sawada et al. 1999; Shrikant et al. 1995) and in vivo in both adult and postnatal models of injury (Balasingam and Yong 1996; Jackson et al. 2005; Ooboshi et al. 2005; Pang et al. 2005). 
opening the possibility that IL-10-mediated effects on microglial response reported here depend on the earlier microglial induction by NLSCt.

\section{NLSCt induced an early increase in IL-1 $\beta$, but IL-10 did not modulate pro-inflammatory cytokines.}

We here report that, concomitantly with its neuroprotective action, NLSCt administration induced an early and transient increase in IL-1 $\beta$ production at $12 \mathrm{hpl}$, correlating with the increase in the density of microglia/macrophages, the main cell type expressing IL-1 $\beta$ in this model (Acarin et al. 2000; Acarin et al. 2002). This increase in IL-1 $\beta$ production is somewhat surprising, as IL-1 $\beta$ has been classically considered to be deleterious on lesion outcome, both in the adult (Rothwell 2003) and postnatal brain (Cai et al. 2004; Hagan et al. 1996; Hagberg et al. 1996).

Otherwise, it should be noted that the IL-10 mediated modulation of astroglial and microglial cell responses was not associated with overall changes in the production of the main pro-inflammatory cytokines, produced by these glial populations (Acarin et al. 2000). IL-1ß and TNF $\alpha$ were not affected, and only a mild increase in late IL-6 production was seen. However, reports on adult animals and in vitro studies, have demonstrated that IL-10 is a strong inhibitor of IL-1 $\beta$, TNF $\alpha$ and IL-6 (Benveniste et al. 1995; Knoblach and Faden 1998; Ledeboer et al. 2000; Lodge and Sriram 1996; Molina-Holgado et al. 2001b; Ooboshi et al. 2005; Pousset et al. 1999; Sawada et al. 1999), key factors in the initiation and propagation of CNS inflammation (Allan and Rothwell 2001; Gadient and Otten 1997; Rothwell 2003; Shohami et al. 1999; Touzani et al. 1999). These observations further indicate the existence of age-related differences in the response of glial cells to IL-10, and/or in the inhibitory role of IL-10 on their pro-inflammatory cytokine production. The latter is further supported by the fact that peripheral blood monocytes, which constitute microglial precursors during development (Cuadros and Navascues 1998; Kaur et al. 2001), are less responsive to IL-10-mediated inhibition of pro-inflammatory cytokine production when they are obtained from newborns as compared to those isolated from adults (Schultz et al. 2004).

In conclusion, this study shows that the combined use of IL-10 overexpression together with the RGDinteracting vector NLSCt did not synergistically exert a neuroprotective action, instead, IL-10 overexpression was deleterious for excitotoxic damage progression, including exacerbation of neurodegeneration, neutrophil recruitment and microglia/macrophage response, which occurred concomitant with a downregulation in the astroglial response and no overall changes in inflammatory cytokine production. 


\section{ACKNOWLEDGMENTS}

We would like to thank Miguel Angel Martil, Maria Agustina Gonzalez and Dolores Mulero for their excellent technical help. 


\section{REFERENCES}

Abraham KE, McMillen D, Brewer KL. 2004. The effects of endogenous interleukin-10 on gray matter damage and the development of pain behaviors following excitotoxic spinal cord injury in the mouse. Neuroscience 124(4):945-952.

Acarin L, Gonzalez B, Castellano B. 2000. Neuronal, astroglial and microglial cytokine expression after an excitotoxic lesion in the immature rat brain. Eur J Neurosci 12(10):3505-3520.

Acarin L, Gonzalez B, Castellano B. 2001. Triflusal posttreatment inhibits glial nuclear factor-kappaB, downregulates the glial response, and is neuroprotective in an excitotoxic injury model in postnatal brain. Stroke 32(10):23942402.

Acarin L, Gonzalez B, Castellano B, Castro AJ. 1996. Microglial response to N-methyl-D-aspartate-mediated excitotoxicity in the immature rat brain. J Comp Neurol 367(3):361-374.

Acarin L, Gonzalez B, Castellano B, Castro AJ. 1997. Quantitative analysis of microglial reaction to a cortical excitotoxic lesion in the early postnatal brain. Exp Neurol 147(2):410-417.

Acarin L, Gonzalez B, Castro AJ, Castellano B. 1999a. Primary cortical glial reaction versus secondary thalamic glial response in the excitotoxically injured young brain: microglial/macrophage response and major histocompatibility complex class I and II expression. Neuroscience 89(2):549-565.

Acarin L, Gonzalez B, Hidalgo J, Castro AJ, Castellano B. 1999b. Primary cortical glial reaction versus secondary thalamic glial response in the excitotoxically injured young brain: astroglial response and metallothionein expression. Neuroscience 92(3):827-839.

Acarin L, Peluffo H, Gonzalez B, Castellano B. 2002. Expression of inducible nitric oxide synthase and cyclooxygenase-2 after excitotoxic damage to the immature rat brain. J Neurosci Res 68(6):745-754.

Acarin L, Vela JM, Gonzalez B, Castellano B. 1994. Demonstration of poly-N-acetyl lactosamine residues in ameboid and ramified microglial cells in rat brain by tomato lectin binding. J Histochem Cytochem 42(8): 1033-1041.

Agnello D VP, Ghezzi P. 2000. Increased tumor necrosis factor and interleukin-6 production in the central nervous system of interleukin-10-deficient mice. Brain Res 869(1-2):241-243.

Allan SM, Rothwell NJ. 2001. Cytokines and acute neurodegeneration. Nat Rev Neurosci 2(10):734-744.

Anderova M, Kubinova S, Mazel T, Chvatal A, Eliasson C, Pekny M, Sykova E. 2001. Effect of elevated K(+), hypotonic stress, and cortical spreading depression on astrocyte swelling in GFAP-deficient mice. Glia 35(3):189-203.

Apelt J, Schliebs R. 2001. Beta-amyloid-induced glial expression of both pro- and anti-inflammatory cytokines in cerebral cortex of aged transgenic Tg2576 mice with Alzheimer plaque pathology. Brain Res 894(1):21-30.

Aris A, Villaverde A. 2003. Engineering nuclear localization signals in modular protein vehicles for gene therapy. Biochem Biophys Res Commun 304(4):625-631.

Arvin KL, Han BH, Du Y, Lin SZ, Paul SM, Holtzman DM. 2002. Minocycline markedly protects the neonatal brain against hypoxic-ischemic injury. Ann Neurol 52(1):54-61.

Balasingam V, Tejada-Berges T, Wright E, Bouckova R, Yong VW. 1994. Reactive astrogliosis in the neonatal mouse brain and its modulation by cytokines. J Neurosci 14(2):846-856.

Balasingam V, Yong VW. 1996. Attenuation of astroglial reactivity by interleukin-10. J Neurosci 16(9):2945-2955.

Benveniste EN, Tang LP, Law RM. 1995. Differential regulation of astrocyte TNF-alpha expression by the cytokines TGF-beta, IL-6 and IL-10. Int J Dev Neurosci 13(3-4):341-349.

Breckwoldt MO, Chen JW, Stangenberg L, Aikawa E, Rodriguez E, Qiu S, Moskowitz MA, Weissleder R. 2008. Tracking the inflammatory response in stroke in vivo by sensing the enzyme myeloperoxidase. Proc Natl Acad Sci U S A 105(47):18584-18589.

Brewer KL, Bethea JR, Yezierski RP. 1999. Neuroprotective effects of interleukin-10 following excitotoxic spinal cord injury. Exp Neurol 159(2):484-493.

Buchwald UK, Geerdes-Fenge HF, Vockler J, Ziege S, Lode H. 1999. Interleukin-10: effects on phagocytosis and adhesion molecule expression of granulocytes and monocytes in a comparison with prednisolone. Eur J Med Res 4(3):85-94.

Buisson A, Lesne S, Docagne F, Ali C, Nicole O, MacKenzie ET, Vivien D. 2003. Transforming growth factor-beta and ischemic brain injury. Cell Mol Neurobiol 23(4-5):539-550.

Bush TG, Puvanachandra N, Horner CH, Polito A, Ostenfeld T, Svendsen CN, Mucke L, Johnson MH, Sofroniew MV. 1999. Leukocyte infiltration, neuronal degeneration, and neurite outgrowth after ablation of scar-forming, reactive astrocytes in adult transgenic mice. Neuron 23(2):297-308.

Cai Z, Lin S, Pang Y, Rhodes PG. 2004. Brain injury induced by intracerebral injection of interleukin-1beta and tumor necrosis factor-alpha in the neonatal rat. Pediatr Res 56(3):377-384.

Chen CC, Manning AM. 1996. TGF-beta 1, IL-10 and IL-4 differentially modulate the cytokine-induced expression of IL-6 and IL-8 in human endothelial cells. Cytokine 8(1):58-65.

Chew LJ, Takanohashi A, Bell M. 2006. Microglia and inflammation: impact on developmental brain injuries. Ment Retard Dev Disabil Res Rev 12(2):105-112.

Cornette L. 2004. Fetal and neonatal inflammatory response and adverse outcome. Semin Fetal Neonatal Med 9(6):459470 . 
Cuadros MA, Navascues J. 1998. The origin and differentiation of microglial cells during development. Prog Neurobiol 56(2):173-189.

Dammann O, Leviton A. 1997. Maternal intrauterine infection, cytokines, and brain damage in the preterm newborn. Pediatr Res 42(1):1-8.

Dietrich WD, Busto R, Bethea JR. 1999. Postischemic hypothermia and IL-10 treatment provide long-lasting neuroprotection of CA1 hippocampus following transient global ischemia in rats. Exp Neurol 158(2):444-450.

Ehrlich LC, Hu S, Peterson PK, Chao CC. 1998. IL-10 down-regulates human microglial IL-8 by inhibition of NFkappaB activation. Neuroreport 9(8):1723-1726.

Fan LW, Pang Y, Lin S, Rhodes PG, Cai Z. 2005. Minocycline attenuates lipopolysaccharide-induced white matter injury in the neonatal rat brain. Neuroscience 133(1):159-168.

Faulkner JR, Herrmann JE, Woo MJ, Tansey KE, Doan NB, Sofroniew MV. 2004. Reactive astrocytes protect tissue and preserve function after spinal cord injury. J Neurosci 24(9):2143-2155.

Ferriero DM. 2004. Neonatal brain injury. N Engl J Med 351(19):1985-1995.

Ferriero DM, Holtzman DM, Black SM, Sheldon RA. 1996. Neonatal mice lacking neuronal nitric oxide synthase are less vulnerable to hypoxic-ischemic injury. Neurobiol Dis 3(1):64-71.

Froen JF, Munkeby BH, Stray-Pedersen B, Saugstad OD. 2002. Interleukin-10 reverses acute detrimental effects of endotoxin-induced inflammation on perinatal cerebral hypoxia-ischemia. Brain Res 942(1-2):87-94.

Gadient RA, Otten UH. 1997. Interleukin-6 (IL-6)--a molecule with both beneficial and destructive potentials. Prog Neurobiol 52(5):379-390.

Galasso JM, Liu Y, Szaflarski J, Warren JS, Silverstein FS. 2000. Monocyte chemoattractant protein-1 is a mediator of acute excitotoxic injury in neonatal rat brain. Neuroscience 101(3):737-744.

Gonzalez FF, Ferriero DM. 2008. Therapeutics for neonatal brain injury. Pharmacol Ther 120(1):43-53.

Gonzalez P, Burgaya F, Acarin L, Peluffo H, Castellano B, Gonzalez B. 2009. Interleukin-10 and Interleukin-10 Receptor-I Are Upregulated in Glial Cells After an Excitotoxic Injury to the Postnatal Rat Brain. J Neuropathol Exp Neurol 68(4):391-403.

Guo H, Jin YX, Ishikawa M, Huang YM, van der Meide PH, Link H, Xiao BG. 1998. Regulation of beta-chemokine mRNA expression in adult rat astrocytes by lipopolysaccharide, proinflammatory and immunoregulatory cytokines. Scand J Immunol 48(5):502-508.

Hagan P, Barks JD, Yabut M, Davidson BL, Roessler B, Silverstein FS. 1996. Adenovirus-mediated over-expression of interleukin-1 receptor antagonist reduces susceptibility to excitotoxic brain injury in perinatal rats. Neuroscience 75(4):1033-1045.

Hagberg H, Gilland E, Bona E, Hanson LA, Hahin-Zoric M, Blennow M, Holst M, McRae A, Soder O. 1996. Enhanced expression of interleukin (IL)-1 and IL-6 messenger RNA and bioactive protein after hypoxia-ischemia in neonatal rats. Pediatr Res 40(4):603-609.

Hamada Y, Hayakawa T, Hattori H, Mikawa H. 1994. Inhibitor of nitric oxide synthesis reduces hypoxic-ischemic brain damage in the neonatal rat. Pediatr Res 35(1):10-14.

Hartl R, Schurer L, Schmid-Schonbein GW, del Zoppo GJ. 1996. Experimental antileukocyte interventions in cerebral ischemia. J Cereb Blood Flow Metab 16(6):1108-1119.

Hudome S, Palmer C, Roberts RL, Mauger D, Housman C, Towfighi J. 1997. The role of neutrophils in the production of hypoxic-ischemic brain injury in the neonatal rat. Pediatr Res 41(5):607-616.

Hughes EG, Maguire JL, McMinn MT, Scholz RE, Sutherland ML. 2004. Loss of glial fibrillary acidic protein results in decreased glutamate transport and inhibition of PKA-induced EAAT2 cell surface trafficking. Brain Res Mol Brain Res 124(2):114-123.

Hulshof S, Montagne L, De Groot CJ, Van Der Valk P. 2002. Cellular localization and expression patterns of interleukin-10, interleukin-4, and their receptors in multiple sclerosis lesions. Glia 38(1):24-35.

Jackson CA, Messinger J, Peduzzi JD, Ansardi DC, Morrow CD. 2005. Enhanced functional recovery from spinal cord injury following intrathecal or intramuscular administration of poliovirus replicons encoding IL-10. Virology 336(2):173-183.

Jander S, Pohl J, D'Urso D, Gillen C, Stoll G. 1998. Time course and cellular localization of interleukin-10 mRNA and protein expression in autoimmune inflammation of the rat central nervous system. Am J Pathol 152(4):975-982.

Kadhim H, Tabarki B, Verellen G, De Prez C, Rona AM, Sebire G. 2001. Inflammatory cytokines in the pathogenesis of periventricular leukomalacia. Neurology 56(10):1278-1284.

Kamm K, Vanderkolk W, Lawrence C, Jonker M, Davis AT. 2006. The effect of traumatic brain injury upon the concentration and expression of interleukin-1beta and interleukin-10 in the rat. J Trauma 60(1):152-157.

Kaur C, Hao AJ, Wu CH, Ling EA. 2001. Origin of microglia. Microsc Res Tech 54(1):2-9.

Kline AE, Bolinger BD, Kochanek PM, Carlos TM, Yan HQ, Jenkins LW, Marion DW, Dixon CE. 2002. Acute systemic administration of interleukin-10 suppresses the beneficial effects of moderate hypothermia following traumatic brain injury in rats. Brain Res 937(1-2):22-31.

Knoblach SM, Faden AI. 1998. Interleukin-10 improves outcome and alters proinflammatory cytokine expression after experimental traumatic brain injury. Exp Neurol 153(1):143-151.

Kochanek PM, Hallenbeck JM. 1992. Polymorphonuclear leukocytes and monocytes/macrophages in the pathogenesis of cerebral ischemia and stroke. Stroke 23(9):1367-1379. 
Krakauer T. 1995. IL-10 inhibits the adhesion of leukocytic cells to IL-1-activated human endothelial cells. Immunol Lett 45(1-2):61-65.

Kremlev SG, Roberts RL, Palmer C. 2007. Minocycline modulates chemokine receptors but not interleukin-10 mRNA expression in hypoxic-ischemic neonatal rat brain. J Neurosci Res 85(11):2450-2459.

Lawson LJ, Perry VH. 1995. The unique characteristics of inflammatory responses in mouse brain are acquired during postnatal development. Eur J Neurosci 7(7):1584-1595.

Ledeboer A, Breve JJ, Poole S, Tilders FJ, Van Dam AM. 2000. Interleukin-10, interleukin-4, and transforming growth factor-beta differentially regulate lipopolysaccharide-induced production of pro-inflammatory cytokines and nitric oxide in co-cultures of rat astroglial and microglial cells. Glia 30(2):134-142.

Ledeboer A, Wierinckx A, Bol JG, Floris S, Renardel de Lavalette C, De Vries HE, van den Berg TK, Dijkstra CD, Tilders FJ, van dam AM. 2003. Regional and temporal expression patterns of interleukin-10, interleukin-10 receptor and adhesion molecules in the rat spinal cord during chronic relapsing EAE. J Neuroimmunol 136(12):94-103.

Leviton A, Dammann O. 2004. Coagulation, inflammation, and the risk of neonatal white matter damage. Pediatr Res 55(4):541-545.

Liberto CM, Albrecht PJ, Herx LM, Yong VW, Levison SW. 2004. Pro-regenerative properties of cytokine-activated astrocytes. J Neurochem 89(5):1092-1100.

Lin S, Cox HJ, Rhodes PG, Cai Z. 2006. Neuroprotection of alpha-phenyl-n-tert-butyl-nitrone on the neonatal white matter is associated with anti-inflammation. Neurosci Lett 405(1-2):52-56.

Lodge PA, Sriram S. 1996. Regulation of microglial activation by TGF-beta, IL-10, and CSF-1. J Leukoc Biol $\operatorname{Oct}(60): 4$.

Mesples B, Fontaine RH, Lelievre V, Launay JM, Gressens P. 2005. Neuronal TGF-beta1 mediates IL-9/mast cell interaction and exacerbates excitotoxicity in newborn mice. Neurobiol Dis 18(1):193-205.

Mesples B, Plaisant F, Gressens P. 2003. Effects of interleukin-10 on neonatal excitotoxic brain lesions in mice. Brain Res Dev Brain Res 141(1-2):25-32.

Molina-Holgado E, Arevalo-Martin A, Castrillo A, Bosca L, Vela JM, Guaza C. 2002a. Interleukin-4 and interleukin-10 modulate nuclear factor kappaB activity and nitric oxide synthase-2 expression in Theiler's virus-infected brain astrocytes. J Neurochem 81(6):1242-1252.

Molina-Holgado E, Arevalo-Martin A, Ortiz S, Vela JM, Guaza C. 2002b. Theiler's virus infection induces the expression of cyclooxygenase- 2 in murine astrocytes: inhibition by the anti-inflammatory cytokines interleukin-4 and interleukin-10. Neurosci Lett 324(3):237-241.

Molina-Holgado E, Vela JM, Arevalo-Martin A, Guaza C. 2001a. LPS/IFN-gamma cytotoxicity in oligodendroglial cells: role of nitric oxide and protection by the anti-inflammatory cytokine IL-10. Eur J Neurosci 13(3):493502 .

Molina-Holgado F, Grencis R, Rothwell NJ. 2001b. Actions of exogenous and endogenous IL-10 on glial responses to bacterial LPS/cytokines. Glia 33(2):97-106.

Moore KW, de Waal Malefyt R, Coffman RL, O'Garra A. 2001. Interleukin-10 and the interleukin-10 receptor. Annu Rev Immunol 19:683-765.

Mostafa Mtairag E, Chollet-Martin S, Oudghiri M, Laquay N, Jacob MP, Michel JB, Feldman LJ. 2001. Effects of interleukin-10 on monocyte/endothelial cell adhesion and MMP-9/TIMP-1 secretion. Cardiovasc Res 49(4):882-890.

Myer DJ, Gurkoff GG, Lee SM, Hovda DA, Sofroniew MV. 2006. Essential protective roles of reactive astrocytes in traumatic brain injury. Brain 129(Pt 10):2761-2772.

Nawashiro H, Brenner M, Fukui S, Shima K, Hallenbeck JM. 2000. High susceptibility to cerebral ischemia in GFAPnull mice. J Cereb Blood Flow Metab 20(7):1040-1044.

Nawashiro H, Messing A, Azzam N, Brenner M. 1998. Mice lacking GFAP are hypersensitive to traumatic cerebrospinal injury. Neuroreport 9(8):1691-1696.

Northington FJ. 2006. Brief update on animal models of hypoxic-ischemic encephalopathy and neonatal stroke. Ilar J 47(1):32-38.

O'Keefe GM, Nguyen VT, Benveniste EN. 1999. Class II transactivator and class II MHC gene expression in microglia: modulation by the cytokines TGF-beta, IL-4, IL-13 and IL-10. Eur J Immunol 29(4):1275-1285.

Ooboshi H, Ibayashi S, Shichita T, Kumai Y, Takada J, Ago T, Arakawa S, Sugimori H, Kamouchi M, Kitazono T, Iida M. 2005. Postischemic gene transfer of interleukin-10 protects against both focal and global brain ischemia. Circulation 111(7):913-919.

Palmer C, Roberts RL, Young PI. 2004. Timing of neutrophil depletion influences long-term neuroprotection in neonatal rat hypoxic-ischemic brain injury. Pediatr Res 55(4):549-556.

Pang Y, Rodts-Palenik S, Cai Z, Bennett WA, Rhodes PG. 2005. Suppression of glial activation is involved in the protection of IL-10 on maternal E. coli induced neonatal white matter injury. Brain Res Dev Brain Res 157(2):141-149.

Pekny M, Stanness KA, Eliasson C, Betsholtz C, Janigro D. 1998. Impaired induction of blood-brain barrier properties in aortic endothelial cells by astrocytes from GFAP-deficient mice. Glia 22(4):390-400. 
Peluffo H, Acarin L, Aris A, Gonzalez P, Villaverde A, Castellano B, Gonzalez B. 2006. Neuroprotection from NMDA excitotoxic lesion by $\mathrm{Cu} / \mathrm{Zn}$ superoxide dismutase gene delivery to the postnatal rat brain by a modular protein vector. BMC Neurosci 7(1):35.

Peluffo H, Acarin L, Faiz M, Castellano B, Gonzalez B. 2005. Cu/Zn Superoxide dismutase expression in the postnatal rat brain following an excitotoxic injury. J Neuroinflammation 2(1):12.

Peluffo H, Aris A, Acarin L, Gonzalez B, Villaverde A, Castellano B. 2003. Nonviral gene delivery to the central nervous system based on a novel integrin-targeting multifunctional protein. Hum Gene Ther 14(13):1215-1223.

Peluffo H, Gonzalez P, Aris A, Acarin L, Saura J, Villaverde A, Castellano B, Gonzalez B. 2007. RGD domains neuroprotect the immature brain by a glial-dependent mechanism. Ann Neurol 62(3):251-261.

Pousset F, Cremona S, Dantzer R, Kelley K, Parnet P. 1999. Interleukin-4 and interleukin-10 regulate IL1-beta induced mouse primary astrocyte activation: a comparative study. Glia 26(1):12-21.

Pousset F, Cremona S, Dantzer R, Kelley KW, Parnet P. 2001. IL-10 and IL-4 regulate type-I and type-II IL-1 receptors expression on IL-1 beta-activated mouse primary astrocytes. J Neurochem 79(4):726-736.

Rezaie P, Dean A. 2002. Periventricular leukomalacia, inflammation and white matter lesions within the developing nervous system. Neuropathology 22(3):106-132.

Rodts-Palenik S, Wyatt-Ashmead J, Pang Y, Thigpen B, Cai Z, Rhodes P, Martin JN, Granger J, Bennett WA. 2004. Maternal infection-induced white matter injury is reduced by treatment with interleukin-10. Am J Obstet Gynecol 191(4):1387-1392.

Rothwell N. 2003. Interleukin-1 and neuronal injury: mechanisms, modification, and therapeutic potential. Brain Behav Immun 17(3):152-157.

Sawada M, Suzumura A, Hosoya H, Marunouchi T, Nagatsu T. 1999. Interleukin-10 inhibits both production of cytokines and expression of cytokine receptors in microglia. J Neurochem 72(4):1466-1471.

Schultz C, Temming P, Bucsky P, Gopel W, Strunk T, Hartel C. 2004. Immature anti-inflammatory response in neonates. Clin Exp Immunol 135(1):130-136.

Shohami E, Ginis I, Hallenbeck JM. 1999. Dual role of tumor necrosis factor alpha in brain injury. Cytokine Growth Factor Rev 10(2):119-130.

Shrikant P, Weber E, Jilling T, Benveniste EN. 1995. Intercellular adhesion molecule-1 gene expression by glial cells. Differential mechanisms of inhibition by IL-10 and IL-6. J Immunol 155(3):1489-1501.

Silverstein FS, Barks JD, Hagan P, Liu XH, Ivacko J, Szaflarski J. 1997. Cytokines and perinatal brain injury. Neurochem Int 30(4-5):375-383.

Smith PK, Krohn RI, Hermanson GT, Mallia AK, Gartner FH, Provenzano MD, Fujimoto EK, Goeke NM, Olson BJ, Klenk DC. 1985. Measurement of protein using bicinchoninic acid. Anal Biochem 150(1):76-85.

Spera PA, Ellison JA, Feuerstein GZ, Barone FC. 1998. IL-10 reduces rat brain injury following focal stroke. Neurosci Lett 251(3):189-192.

Strle K, Zhou JH, Shen WH, Broussard SR, Johnson RW, Freund GG, Dantzer R, Kelley KW. 2001. Interleukin-10 in the brain. Crit Rev Immunol 21(5):427-449.

Tahraoui SL, Marret S, Bodenant C, Leroux P, Dommergues MA, Evrard P, Gressens P. 2001. Central role of microglia in neonatal excitotoxic lesions of the murine periventricular white matter. Brain Pathol 11(1):56-71.

Touzani O, Boutin H, Chuquet J, Rothwell N. 1999. Potential mechanisms of interleukin-1 involvement in cerebral ischaemia. J Neuroimmunol 100(1-2):203-215.

Tsuji M, Higuchi Y, Shiraishi K, Kume T, Akaike A, Hattori H. 2000. Protective effect of aminoguanidine on hypoxicischemic brain damage and temporal profile of brain nitric oxide in neonatal rat. Pediatr Res 47(1):79-83.

Vannucci SJ, Hagberg H. 2004. Hypoxia-ischemia in the immature brain. J Exp Biol 207(Pt 18):3149-3154.

Woiciechowsky C, Schoning B, Stoltenburg-Didinger G, Stockhammer F, Volk HD. 2004. Brain-IL-1 beta triggers astrogliosis through induction of IL-6: inhibition by propranolol and IL-10. Med Sci Monit 10(9):BR325-330.

Zhai QH, Futrell N, Chen FJ. 1997. Gene expression of IL-10 in relationship to TNF-alpha, IL-1beta and IL-2 in the rat brain following middle cerebral artery occlusion. J Neurol Sci 152(2):119-124. 


\section{FIGURE LEGENDS}

Figure 1. Schematic representation of areas selected for densitometry and cell count analysis. Schematic drawings of coronal sections at three different bregma levels, showing areas selected for densitometrical analysis of immunoreactivity and cell counting. Gray area in each section indicates extension of tissue damage, and the arrow points to the intracortical injection site of NMDA and NLSCt carrying either GFP or IL-10 transgenes. Densitometrical analysis and cell counts in the lesion center were performed in $0.18 \mathrm{~mm}^{2}$ images taken at $20 \mathrm{x}$, whereas cell counts in the periphery of the lesion were carried out in two adjacent $0.04 \mathrm{~mm}^{2}$ images taken at $40 \mathrm{x}$. Scale bar $=1 \mathrm{~mm}$.

Figure 2. IL-10 expression. Figure shows data obtained from IL-10 expression quantification by Real Time PCR at 24 hours post-lesion. Note that NMDA/NLSCt/IL-10 animals displayed a significant increase in IL-10 expression in comparison with both NMDA/NLSCt/GFP and NMDA/SAL control groups.

Figure 3. Analysis of lesion volume. Figure shows representative Nissl stained sections used for lesion volume quantification from each experimental group and survival time. Area of tissue injury is delimited by black lines. Data showing lesion volume quantification is represented in the lower graph. NMDA/NLSCt/IL-10 animals showed increased lesion volume at $12 \mathrm{hpl}$ in comparison with both control groups, and at $72 \mathrm{hpl}$ when compared to the NMDA/NLSCt/GFP group. Statistically significant differences are shown as: *, when compared to NMDA/SAL; \#, when compared to NMDA/NLSCt/GFP; \&, in relation to the same group at the previous survival time. DPL, days postlesion.

Figure 4. Evaluation of neuronal degeneration. Quantitative analysis of Fluoro-Jade B (FJB) staining for degenerating neurons is shown in A; B-J show representative micrographs corresponding to the different experimental groups and survival times analyzed. NMDA/NLSCt/IL-10 animals displayed increased density of FJB+ degenerating neurons at $12 \mathrm{hpl}$ in comparison with both control groups and, at 72hpl, when compared to the NMDA/NLSCt/GFP group. Statistically significant differences in A are shown as: *, when compared to NMDA/SAL; \#, when compared to NMDA/NLSCt/GFP; \&, in relation to the same group at the previous survival time. HPL, hours post-lesion; DPL, days post-lesion. Scale bars $=20 \mu \mathrm{m}$.

Figure 5. Microglia/macrophage response. Densitometric analysis of tomato lectin (TL) processed sections (A) and quantitative analysis of TL+ microglia/macrophage cell density (B) show an augmented immunoreactivity grade and microglia/macrophage cell density in NMDA/NLSCt/GFP and NMDA/NLSCt/IL-10 groups at 12hpl. Note that both parameters are further increased at $72 \mathrm{hpl}$ only in NMDA/NLSCt/IL-10 animals. C-K show representative images from the different experimental groups and survival times analyzed. Statistically significant differences in A and B are shown as: *, when compared to NMDA/SAL; \#, when compared to NMDA/NLSCt/GFP; \&, in comparison with same group at the previous survival time. HPL, hours post-lesion; DPL, days post-lesion. Scale bars $=20 \mu \mathrm{m}$. 
Figure 6. Analysis of microglial proliferation. Representative micrographs from CD11b/p-H3 double labeled sections from the three experimental groups by 72 hours post-lesion show no differences between groups. Scale bars $=20 \mu \mathrm{m}$.

Figure 7. Densitometrical analysis of astroglial reactivity. Evaluation of the immunoreactivity grade in GFAP processed sections (A) and vimentin processed sections (B) show a significant decrease in both astroglial markers in NMDA/NLSCt/IL-10 animals at $72 \mathrm{hpl}$. Statistically significant differences are shown as: *, when compared to NMDA/SAL; \#, when compared to NMDA/NLSCt/GFP; \&, when comparing the same group at the previous survival time. HPL, hours post-lesion; DPL, days post-lesion.

Figure 8. Microscopic analysis of astroglial reactivity. Micrographs showing representative images of GFAP (A-F) and vimentin (G-L) immunohistochemistry, as the ones used for densitometrical quantification shown in Figure 7. Note the apparent decrease in GFAP (C) and vimentin (I) immunoreactivity in the NMDA/NLSCt/IL-10 group at $72 \mathrm{hpl}$, in comparison to the NMDA/SAL (A and G, respectively) and the NMDA/NLSCt/GFP group (B and H, respectively). Both GFAP and vimentin are increased at $7 \mathrm{dpl}$ in all groups. HPL, hours post-lesion; DPL, days post-lesion. Scale bars $=20 \mu \mathrm{m}$.

Figure 9. Myeloperoxidase (MPO)+ cell analysis. Quantification of MPO+ cell density and representative micrographs of MPO processed sections for the evaluation of neutrophil changes in the different experimental groups at $12 \mathrm{hpl}$, show an increase in MPO+ cells in the NMDA/NLSCt/IL-10 group. Statistically significant differences are shown as: *, when compared with NMDA/SAL, or \#, when compared to NMDA/NLSCt/GFP. Scale bars $=20 \mu \mathrm{m}$.

Figure 10. Evaluation of cytokine concentration by ELISA. Graphic representation of interleukin-1 $\beta$ (IL-1 $\beta$ ), tumor necrosis factor $\alpha(\mathrm{TNF} \alpha)$ and interleukin-6 (IL-6) concentration in ipsilateral damaged hemispheres at different survival times and experimental groups, show a significant increase in IL-1 $\beta$ in both NMDA/NLSCt/IL-10 and NMDA/NLSCt/GFP groups at $12 \mathrm{hpl}$, and a mild increase in IL-6 expression in NMDA/NLSCt/IL-10 animals at $7 \mathrm{dpl}$. Statistically significant differences are shown as: *, when compared to NMDA/SAL; and \& when comparing with the same group at the previous survival time. HPL, hours post-lesion; DPL, days post-lesion. 